\title{
Other Drugs Associated with Osteoporosis
}

Systemic osteoporosis has also been observed in patients receiving various medications:

- High-dose and long-term heparin and warfarin therapy is associated with an increased risk of osteoporosis, and there may be a prolonged effect on the bone after cessation of therapy. The use of low-molecular-weight heparin and of newer antithrombotic agents such as fondaparinux is probably associated with fewer skeletal effects.

- Thiazolidinediones (TZDs) are widely used in the treatment of type 2 diabetes. Administration of rosiglitazone resulted in significant bone loss and suppression of biochemical markers of bone formation. In women, there was a significant increase in the incidence of fractures. This drug class effect is positively associated with duration of treatment; men and women are equally affected.

- Selective serotonin receptor uptake inhibitors (SSRIs) are widely described antidepressants and are associated with reduced bone mass and increased fracture risk. However depression itself may be associated with increased risk of osteoporosis.
- Long-term and high-dose acid-suppressive medications (proton pump inhibitors, PPI, e.g. omeprazole) increase fracture risk, though the mechanism remains unknown. Reduced intestinal calcium absorption seems unlikely to be solely responsible for the development of increased fracture risk within 1 year of starting therapy. Inhibition of the osteoclastic proton pump would be expected to have beneficial effects on bone resorption.

Drugs protecting against osteoporosis:

- In several studies, a significant protective effect of beta-blocker therapy on fracture risk has been reported, although this finding has not been universal.

- In thiazide users, reduced fracture risk and increases in bone density have been reported. Increased renal calcium reabsorption may play a role in these effects.

- Statins inhibit an important enzyme in the mevalonate pathway, thus inhibiting osteoclastic activity. However, benefits on fracture risk and BMD were only small in some studies. 